\title{
Photon statistics in the three-photon hyper-Raman process
}

\author{
L. Sáinz de los Terreros, M. Santos, and P. F. González-Díaz \\ Instituto de Optica, Daza de Valdés, Serrano 121, Madrid 6, Spain \\ (Received 18 July 1984)
}

\begin{abstract}
An exact solution of the master equation for the three-photon hyper-Raman process is obtained by a matrix method. Several cases of the photon statistics are considered and the appearance of photon-antibunching effect and the violation of the classical Cauchy inequality are discussed.
\end{abstract}

\section{INTRODUCTION}

The photon statistics of various nonlinear optical processes has been a subject of increasing interest in recent years. In particular, photon statistics for the usual Raman process has been analyzed theoretically by Simaan in detail. ${ }^{1}$ The similar hyper-Raman (HR) process ${ }^{2}$ has also been studied already by Simaan, ${ }^{3}$ who formulated the master equation of the process giving the solution by the Laplace transform method.

In this paper we consider again the HR process and give the solution of the master equation by a new matrix method similar to that used by Zubairy ${ }^{4}$ for multiphoton absorption processes. Our results complete the previous work by giving rather complete explicit numerical results in a number of cases of interest.

\section{MASTER EQUATION FOR THE FIELD}

We consider a coupled system of a large number of identical two-level atoms and two single modes of the radiation field inside a cavity of volume $V$. We assume the atoms make transitions from the ground state 1 to the upper state 2 by absorbing two photons of frequency $\omega_{1}$ from the pumping mode $l$ and simultaneously emitting one photon of frequency $\omega_{s}$ in the Stokes mode $s$. The thermal populations of the two atomic levels involved in the HR transitions are assumed to be maintained constant during the interaction with the radiation field. The interaction Hamiltonian for this process is expressed in the second-quantization formalism by

$H_{\mathrm{int}}=\eta \sum_{i} c_{2 i}^{\dagger} c_{1 i} E_{l}^{(+)}\left(r_{i}\right) E_{l}^{(+)}\left(r_{i}\right) E_{s}^{(-)}\left(r_{i}\right)+$ H.c.

where

$$
\begin{aligned}
E_{k}^{(+)}(r) & =i\left(2 \pi \hbar \omega_{k}\right)^{1 / 2} u_{k}(r) a_{k} \\
& =\left[E_{k}^{(-)}(r)\right]^{\dagger}
\end{aligned}
$$

is the positive-frequency part for the radiation electricfield operator in the $k$ mode, the $a_{k}^{\dagger}, a_{k}$ being the associated creation-destruction photon operators, and $u_{k}(r)$ and its complex conjugated, normalized spatial-mode functions. The $c_{1 i}^{\dagger}, c_{1 i}$, etc., are atomic creation-destruction operators for the corresponding states of the $i$ th atom, and $\eta$ is the effective dipole-matrix element for the transitions.
In the interaction picture, the equation of motion for the density operator $\rho$ of the coupled atoms-field system is given by the Liouville equation

$$
i \hbar \frac{\partial}{\partial t} \rho=\left[H_{\mathrm{int}}(t), \rho(t)\right] \text {. }
$$

We are interested in the field subsystem only so we define the reduced density matrix for it:

$$
\rho_{f}(t)=\operatorname{tr}_{\mathrm{at}} \rho(t)=\sum_{A}\langle A|\rho(t)| A\rangle .
$$

It is assumed that at time $t=0$, when the interaction is switched on, the radiation field and the ensemble of atoms are decoupled, and so

$$
\rho(0)=\rho_{f}(0) \otimes \prod_{i} \rho_{i}(0),
$$

where $\rho_{i}(0)$ is the thermal equilibrium density matrix for the $i$ th atom. By the well-known density matrix timeperturbation techniques, ${ }^{6}$ and invoking the irreversible and Markov approximations, we obtain finally the following equation of motion for the reduced density operator $\rho_{f}$ in the interaction picture:

$$
\begin{gathered}
\frac{\partial}{\partial t} \rho_{f}=-\beta\left[p _ { 1 } \left(a_{s} a_{l}^{\dagger} a_{l}^{\dagger} a_{l} a_{l} a_{s}^{\dagger} \rho_{f}-2 a_{s}^{\dagger} a_{l} a_{l} \rho_{f} a_{s} a_{l}^{\dagger} a_{l}^{\dagger}\right.\right. \\
\left.+\rho_{f} a_{s} a_{l}^{\dagger} a_{l}^{\dagger} a_{s}^{\dagger} a_{l} a_{l}\right) \\
+p_{2}\left(\rho_{f} a_{s}^{\dagger} a_{l} a_{l} a_{s} a_{l}^{\dagger} a_{l}^{\dagger}-2 a_{s} a_{l}^{\dagger} a_{l}^{\dagger} \rho_{f} a_{s}^{\dagger} a_{l} a_{l}\right. \\
\left.\left.+a_{s}^{\dagger} a_{l} a_{l} a_{s} a_{l}^{\dagger} a_{l}^{\dagger} \rho_{f}\right)\right]
\end{gathered}
$$

where

$$
\beta=8 \pi^{4} \hbar \omega_{l}^{2} \omega_{s} g(\Omega)|\eta|^{2} \sum_{i}\left|u_{l}\left(r_{i}\right)\right|^{4}\left|u_{s}\left(r_{i}\right)\right|^{2}
$$

and $g(\Omega)$ is the line function for the HR transitions calculated at resonance, i.e., $\Omega=2 \omega_{l}-\omega_{s}$. Equation (6) is the master equation for the field in which $p_{1}, p_{2}$ denotes the populations of the atomic levels, and $\beta$, whose dimension is (time) $)^{-1}$ is obviously related with the rate at which changes take place in the statistical properties of the field. 


\section{MASTER EQUATION IN THE FOCK SPACE: SHORT-TIME SOLUTIONS}

Equation (6) is the master equation for the HR process in operator form. Taking the diagonal matrix elements in the Fock space of photon-number states $|n, m\rangle$ for the two modes in both sides of this equation, we obtain

$$
\begin{aligned}
\frac{d}{d t} P_{n, m}(t)=-J\left\{p _ { 1 } \left[n(n-1)(m+1) P_{n, m}\right.\right. \\
\left.-(n+1)(n+2) m P_{n+2, m-1}\right] \\
+p_{2}\left[(n+1)(n+2) m P_{n, m}\right. \\
\cdot \\
\left.\left.-n(n-1)(m+1) P_{n-2, m+1}\right]\right\},
\end{aligned}
$$

where $J=2 \beta$ and $P_{n, m}=\left\langle n, m\left|\rho_{f}\right| n, m\right\rangle$ are the diagonal elements of the field density matrix in the photonnumber representation, and measure the joint probability that $n$ photons in the pumping mode and $m$ photons in the Stokes mode are simultaneously present at time $t$. Equation (7) governs the temporal evolution of the statistical properties of the photon field. The significance of Eq. (7) can be made more intuitive in terms of "transition rates" in the space of photon-number states $(n, m)$ of the field, ${ }^{7}$ as shown in Fig. 1: the direct HR transitions $(n, m) \rightarrow(n-2, m+1)$ proceed at a rate $J p_{1} n(n$ $-1)(m+1) P_{n, m}$ while the inverse. HR transitions $(n, m) \rightarrow(n+2, m-1)$ do so at a rate $J p_{2}(n+1)(n$ $+2) m P_{n, m}$. These last transitions depend upon the population of the upper state $p_{2}$, and disappear if $p_{2}=0$, in which case the master equation for the diagonal matrix elements reduces to the form

$$
\begin{aligned}
\frac{d}{d t} P_{n, m}(t)= & -J n(n-1)(m+1) P_{n, m} \\
& +J(n+1)(n+2) m P_{n+2, m-1} .
\end{aligned}
$$

The first-order moments $\langle n(t)\rangle=\sum_{n, m} n P_{n, m}(t)$ and $\langle m(t)\rangle=\sum_{n, m} m P_{n, m}(t)$ are the average values of the number of photons in either mode. Taking the time derivative of these expressions and substituting Eq. (7) into them, we find

$$
\begin{aligned}
\frac{d}{d t}\langle n\rangle=-2 J\left[p_{1}\langle n(n-1)(m+1)\rangle\right. \\
\left.-p_{2}\langle(n+1)(n+2) m\rangle\right], \\
\frac{d}{d t}\langle m\rangle=J\left[p_{1}\langle n(n-1)(m+1)\rangle\right. \\
\left.-p_{2}\langle(n+1)(n+2) m\rangle\right] .
\end{aligned}
$$

By summing the last two equations we obtain the conservative law

$$
\frac{d}{d t}(\langle n\rangle+2\langle m\rangle)=0,
$$

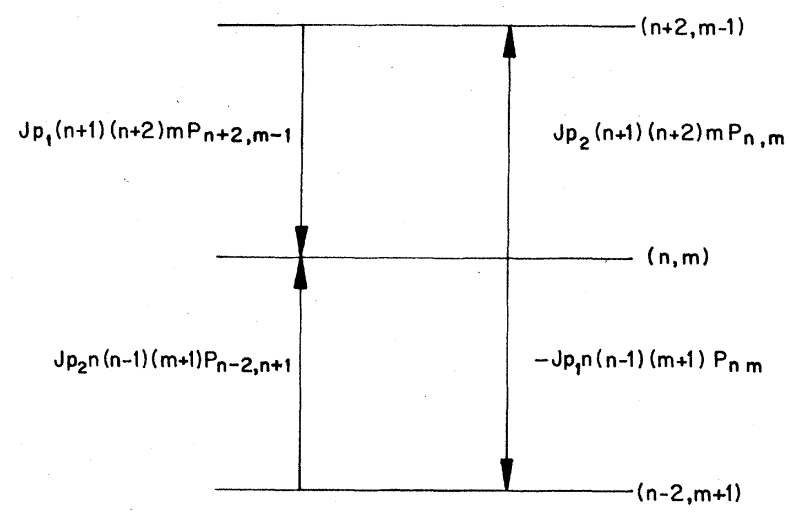

FIG. 1. Transition rates between photon-number states for the three-photon HR process.

which expresses the obvious fact that for each two photons absorbed in the pumping mode, only one photon in the Stokes mode is emitted. We can rearrange easily Eq. (9b) into the equivalent form

$$
\begin{gathered}
\frac{d}{d t}\langle m\rangle=J\left[p_{1}\langle n(n-1)\rangle+\langle n(n-1) m\rangle\left(p_{1}-p_{2}\right)\right. \\
\left.-2\langle(2 n+1) m\rangle p_{2}\right] .
\end{gathered}
$$

The rate at which Stokes photons are created can be expressed as the sum of three contributions. The first term corresponds to the spontaneous effect (when $\langle m\rangle=0$ ). The second term is the stimulated emission term, and it requires the condition $p_{1}>p_{2}$ to be positive. The last term is one of negative gain, as it should be, owing to the presence of inverse HR transitions, in which one photon is annihilated in the Stokes mode while two photons are created in the input mode; this term takes over if $p_{2}=0$. At initial time the pumping and Stokes modes are supposed to be statistically independent, i.e., $P_{n, m}(0)$ $=Q_{n}(0) R_{m}(0)$. It is also assumed that the upper-state population is negligible, $p_{2}=0$, and we can write the initial rate of the process as

$$
\left[\frac{d}{d t}\langle m\rangle\right]_{t=0}=J g_{n_{0}}^{(2)}\left\langle n_{0}\right\rangle^{2}\left(\left\langle m_{0}\right\rangle+1\right),
$$

where we have introduced the second-order coherence degree $g_{n}^{(2)}=\langle n(n-1)\rangle /\langle n\rangle^{2}$. The gain or rate of emission of Stokes photons, defined by

$$
G=J g_{n_{0}}^{(2)}\left\langle n_{0}\right\rangle^{2},
$$

thus depends on the initial statistical properties of the incident light: it is proportional to the square of the intensity (nonlinear effect) and to the degree of second-order coherence. The gain is, therefore, larger in the case of thermal incident radiation $\left(g_{n}^{(2)}=2\right)$ than it would be in the case of coherent incident radiation $\left(g_{n}^{(2)}=1\right)$. This result agrees with the common view that light fluctuations lead to an increase in the rate at which nonlinear processes take place. 
The time derivative of second-order moments $\left\langle n^{2}\right\rangle$ and $\left\langle m^{2}\right\rangle$ and correlation function $\langle n m\rangle$ are easily obtained by a procedure similar to that used for the first-order moments, and we obtain for the initial values of the derivatives,

$$
\begin{gathered}
\left.\qquad \frac{d}{d t}\left\langle m^{2}\right\rangle\right]_{t=0}=J\left\langle n_{0}\right\rangle^{2} g_{n_{0}}^{(2)}\left(2\left\langle m_{0}\right\rangle^{2} g_{m_{0}}^{(2)}\right. \\
\left.+5\left\langle m_{0}\right\rangle+1\right), \\
{\left[\frac{d}{d t}\left\langle n^{2}\right\rangle\right]_{t=0}=-4 J\left(\left\langle n_{0}\right\rangle^{3} g_{n_{0}}^{(3)}+\left\langle n_{0}\right\rangle^{2} g_{n_{0}}^{(2)}\right)} \\
\left.\left.\qquad \frac{d}{d t}\langle n m\rangle\right]_{t=0}=J\left\langle m_{0}\right\rangle+1\right), \\
\left.-2\left\langle m_{0}^{2}\right\rangle^{2} g_{n_{0}}^{(2)} g_{m_{0}}^{(2)}\right\rangle g_{n_{0}}^{(3)}\left(\left\langle m_{0}\right\rangle+1\right) \\
\left.-4\left\langle m_{0}\right\rangle g_{n_{0}}^{(2)}\right]
\end{gathered}
$$

where the right-hand side (rhs) has been expressed in terms of first-order moments and second- and third-order coherence degrees. These are useful intermediate results for calculating the initial time rate for the common statistical functions of the photon fields, and so, to analyze the short-time behavior of these functions up to the linear terms in time. A rather lengthy but straightforward calculation leads us to the values of the initial rates for the second-order coherence degrees,

$$
\begin{gathered}
\left.\int \frac{d}{d t} g_{n}^{(2)}\right]_{t=0}=J\left(\left\langle m_{0}\right\rangle+1\right)\left(4 g_{n_{0}}^{(2) 2}\left\langle n_{0}\right\rangle-2 g_{n_{0}}^{(2)}\right. \\
\left.-4\left\langle n_{0}\right\rangle g_{n_{0}}^{(3)}\right)
\end{gathered}
$$

$$
\left[\frac{d}{d t} g_{m}^{(2)}\right]_{t=0}=2 J\left(\left\langle n_{0}\right\rangle^{2} /\left\langle m_{0}\right\rangle\right) g_{n_{0}}^{(2)}\left(2-g_{m_{0}}^{(2)}\right)
$$

and the correlation degree, defined by $g_{c}=\langle n m\rangle /$ $\langle n\rangle\langle m\rangle$,

$$
\left[\frac{d}{d t} g_{c}\right]_{t=0}=J\left(\left\langle n_{0}\right\rangle /\left\langle m_{0}\right\rangle\right)\left[\left(2\left\langle m_{0}\right\rangle^{2}-\left\langle n_{0}\right\rangle-2\left\langle m_{0}\right\rangle-\left\langle n_{0}\right\rangle\left\langle m_{0}\right\rangle\right) g_{n_{0}}^{(2)}+\left\langle n_{0}\right\rangle\left(\left\langle m_{0}\right\rangle+1\right) g_{n_{0}}^{(3)}-2\left\langle m_{0}\right\rangle^{2} g_{n_{0}}^{(2)} g_{m_{0}}^{(2)}\right]
$$

Equations (14) can be used to predict the behavior in time of the statistical functions of the field at the beginning of the process. We note that the second-order coherence degree of the Stokes mode does not change in first approximation if it is initially chaotic, i.e., $(d / d t)_{t=0} g_{m}^{(2)}=0$ if $g_{m_{0}}^{(2)}=2$. Also, we note that the change in second-order coherence degree of the pumping mode depends only on its own initial statistical properties, but not on the statistical coherence properties of the Stokes mode. It is worth noting that for completely coherent pumping light Eq. (14a) reduces to $(d / d t)_{t=0} g_{n}^{(2)}=-2 J\left(\left\langle m_{0}\right\rangle+1\right)$, i.e., the initial time rate of $g_{n}^{(2)}$ always takes negative values and does not depend on the initial intensity of the input radiation. An important case is the one in which there are no photons in the Stokes mode at time $t=0$; then Eqs. (14b) and $(14 c)$ break down $\left(\left\langle m_{0}\right\rangle=0,\left\langle m_{0}^{2}\right\rangle=0\right.$, etc.). To overcome this difficulty we must go back to the definition formulas,

$$
g_{m}^{(2)}=\left(\left\langle m^{2}\right\rangle-\langle m\rangle\right) /\langle m\rangle^{2}, g_{c}=\langle n m\rangle /\langle n\rangle\langle m\rangle,
$$

and develop the functions in numerator and denominator up to third order in time. We limit ourselves to the second order in $t$, in which case we can readily determine the limiting values of the functions $g_{m}^{(2)}$ and $g_{c}$ at time $t=0$. Straightforward calculations give

$$
\begin{aligned}
& g_{m_{0}}^{(2)}=\left(2 g_{n_{0}}^{(4)}\right) /\left(g_{n_{0}}^{(2)}\right)^{2} \quad\left(\left\langle m_{0}\right\rangle=0\right), \\
& g_{c_{0}}=\left(g_{n_{0}}^{(3)}\right) /\left(g_{n_{0}}^{(2)}\right) \quad\left(\left\langle m_{0}\right\rangle=0\right),
\end{aligned}
$$

so that for coherent pumping light the second-order coherence degree of the Stokes mode takes the value 2 at time zero, while the correlation degree takes the value 1 (see Fig. 4).

\section{EXACT ANALYTICAL SOLUTION OF THE MASTER EQUATION}

We start with the assumption $p_{2}=0$, i.e., we disregard upward transitions in Fig. 1, and take the master equation in the simplified form of Eq. (8). We write $n+2 m=I$ and use the dimensionless time variable $\tau=J t=2 \beta t$. We then get

$$
\dot{P}_{m}^{(I)}=-b_{m}(I) P_{m}^{(I)}+b_{m-1}(I) P_{m-1}^{(I)},
$$

where we have denoted $P_{I-2 m, m} \equiv P_{m}^{(I)}$ and $b_{m}(I)=(I-2 m)(I-2 m-1)(m+1)$. For the distinct values of $I$ and $m$, these equations can be grouped in "chains" as pictured in Fig. 2. The chains can be labeled with the index $I$ and the number of links for any $I$ is finite; it is clear that $I=2 \alpha$ or $I=2 \alpha+1$ for some integer 


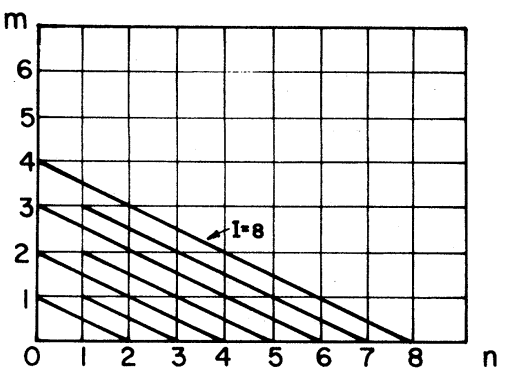

FIG. 2. Rectangular photon-number representation for the two-mode field $(n, m)$. Lattice points represent photon-number states and HR transitions appear as probability flux along the chains $I, n+2 m=I$.

$\alpha \geqq 0$. In this way, index $m$ runs, for a given value of $I$, between 0 and $\alpha(I)=[(I / 2)]$, where the square brackets denote the entire part; the number of links in chain $I$ is therefore $\alpha(I)+1$. We can write, then, Eq. (16) in the form of independent differential matrix systems,

$\left[\begin{array}{c}\dot{P}_{0}^{(I)} \\ \dot{P}_{1}^{(I)} \\ \vdots \\ \dot{P}_{\alpha}^{(I)}\end{array}\right)=\left(\begin{array}{ccccc}-b_{0}(I) & 0 & \cdots & 0 & 0 \\ b_{0}(I) & -b_{1}(I) & \cdots & 0 & 0 \\ \vdots & \vdots & \vdots & \vdots & \vdots \\ 0 & 0 & \cdots & b_{\alpha-1}(I) & -b_{\alpha}(I)\end{array}\right)\left(\begin{array}{c}P_{0}^{(I)} \\ P_{1}^{(I)} \\ \vdots \\ P_{\alpha}^{(I)}\end{array} \mid\right.$,

or in abbreviated matrix form,

$$
\dot{\boldsymbol{P}}^{(I)}=\boldsymbol{M}^{(I)} \boldsymbol{P}^{(I)} .
$$

Each value of $I$ is associated with a particular chain and a corresponding matrix system; we notice in turn several facts.

(1) By summing rows in the two members of Eq. (17) we get $\sum_{m} \dot{P}_{m}^{(I)}=0$, i.e., $\quad \sum_{m} P_{m}^{(I)}(\tau)=\sum_{m} P_{m}^{(I)}(0)$ $=$ const; this is a probability conservation law for each chain $I$.

(2) The matrix $\boldsymbol{M}^{(I)}$ for each system is triangular, so its eigenvalues are the diagonal elements, $\lambda_{m}=-b_{m}(I)$. Hence, the differential system [Eq. (17)] is easily solved analytically by the standard matrix method. It is worth noting that the last eigenvalue in $\boldsymbol{M}^{(I)}$ is always 0; in fact, $\lambda_{\alpha}=-b_{\alpha}(I)=0$ for all $I$. It can be shown that this fact assures the existence of a stationary solution for $\tau \rightarrow \infty$.

(3) A computer-aided calculation of the eigenvalues shows that for certain values of $I$, the corresponding matrix $\boldsymbol{M}^{(I)}$ has some eigenvalue repeated (once) in the diagonal, that is, it has a multiple eigenvalue. Explicitly, it can be shown that for each of the following values of $I$ :

$$
I=10,15,24,25,29,36,38,43, \ldots \text {, }
$$

there corresponds one (and only one) repeated eigenyalue of multiplicity 2; we call the corresponding chains "degenerate."

The general solution of Eq. (17) can be obtained by calculating the corresponding eigenvectors of $\boldsymbol{M}^{(I)}$. We dis- tinguish two cases.

(i) Nondegenerate chains $(I \neq 10,15,24, \ldots)$. In this case, the eigenvalues are simple and the general solution is of the form

$$
\begin{array}{r}
P_{m}^{(I)}(\tau)=\sum_{j=0}^{\alpha} C_{j}(I) A_{j m}(I) \exp \left[-b_{j}(I) \tau\right] \\
(m=0,1, \ldots, \alpha),
\end{array}
$$

where $\mathbf{A}_{j}(I), j=0,1, \ldots, \alpha$ are eigenvectors, corresponding to the eigenvalues $\lambda_{j}=-b_{j}(I)$, respectively, and $C_{j}(I)$ are constants which depend on the initial conditions. The eigenvectors are solutions of the matrix equation

$$
\boldsymbol{M}^{(I)} \mathbf{A}_{j}(I)=-b_{j}(I) \mathbf{A}_{j}(I) .
$$

Solving this matrix equation, we obtain

$$
A_{j m}(I)= \begin{cases}1 & \text { if } m=j \\ 0 & \text { if } m<j \\ m-1 & \frac{b_{k}(I)}{\prod_{k=j}} \quad \text { if } m>j\end{cases}
$$

with $j=0,1, \ldots, \alpha$ and $m=0,1, \ldots, \alpha$.

(ii) Degenerate chains $(I=10,15,24,25,29, \ldots)$. We must take into account the existence of one degenerate eigenvalue with multiplicity 2 , in the diagonal of $\boldsymbol{M}^{(I)}$, i.e., $b_{g}(I)=b_{h}(I)$ for some integers $g<h$. To these multiple eigenvalues there correspond two fundamental solutions which can be written in the following matrix form:

$$
\mathbf{P}_{1,2}^{(I)}=\mathbf{R} \exp [-b(I) \tau]+\mathbf{S} \tau \exp [-b(I) \tau],
$$

where $b(I)=b_{g}(I)=b_{h}(I)$ and $\mathbf{R}$ and $\mathbf{S}$ are unknown column matrices. It can be shown immediately, by substituting these trial solutions into Eq. (17), that $\mathbf{R}$ and $\mathbf{S}$ must satisfy the equations

$$
\begin{aligned}
& \boldsymbol{M}^{(I)} \mathbf{R}=-b(I) \mathbf{R}+\mathbf{S}, \\
& \boldsymbol{M}^{(I)} \mathbf{S}=-b(I) \mathbf{S} .
\end{aligned}
$$

As a first solution we choose $\mathbf{S}=\mathbf{0}, \mathbf{R}=\mathbf{A}_{h}(I)$, so that we can take

$$
\mathbf{P}_{1}^{(I)}(\tau)=\mathbf{A}_{h}(I) \exp [-b(I) \tau] .
$$

A second solution comes by choosing $\mathbf{S}=\mathbf{A}_{h}(I)$, $\mathbf{R}=\mathbf{D}(I)$, and we get

$$
\mathbf{P}_{2}^{(I)}(\tau)=\mathbf{D}(I) \exp [-b(I) \tau]+\mathbf{A}_{h}(I) \tau \exp [-b(I) \tau],
$$

where the column matrix $\mathbf{D}(I)$ must satisfy Eq. (22a), with $\mathbf{S}=\mathbf{A}_{h}(I)$, i.e., $\left[\boldsymbol{M}^{(I)}+\mathbb{1} b(I)\right] \mathbf{D}(I)=\mathbf{A}_{h}(I)$.

The general solution of the master equation can then be expressed as follows:

$$
P_{n, m}(\tau)=\sum_{j=0}^{\alpha(I)} C_{j}(I) A_{j m}(I) \exp \left[-b_{j}(I) \tau\right]
$$

if $n+2 m=I \neq 10,15,24,25,29, \ldots$, and 


$$
\begin{aligned}
P_{n, m}(\tau)=\sum_{\substack{j=0 \\
j \neq g, h}}^{\alpha(I)}\left\{C_{j}(I) A_{j m}(I) \exp \left[-b_{j}(I) \tau\right]\right\} \\
+\kappa(I) A_{h m}(I) \exp [-b(I) \tau] \\
+\iota(I)\left[D_{m}(I)+A_{h m}(I) \tau\right] \exp [-b(I) \tau]
\end{aligned}
$$

if $n+2 m=I=10,15,24,25,29, \ldots$ Formulas for the components of the column matrix $\mathbf{D}(I)$ and the constants $C_{j}(I), \kappa(I)$, and $\iota(I)$ are developed in the Appendix.

The time evolution for the off-diagonal matrix elements $P_{n, m ; n^{\prime}, m^{\prime}}=\left\langle n, m\left|\rho_{f}(\tau)\right| n^{\prime}, m^{\prime}\right\rangle$ is

$$
\begin{aligned}
\frac{d}{d \tau} P_{n, m ; n^{\prime}, m^{\prime}}(\tau)= & -b\left(n, m ; n^{\prime}, m^{\prime}\right) P_{n, m ; n^{\prime}, m^{\prime}} \\
& +c\left(n+2, m-1 ; n^{\prime}+2, m^{\prime}-1\right) \\
& \times P_{n+2, m-1 ; n^{\prime}+2, m^{\prime}-1}
\end{aligned}
$$

where

$$
\begin{aligned}
b\left(n, m ; n^{\prime}, m^{\prime}\right)=\frac{1}{2} & \{n(n-1)(m+1) \\
& \left.+n^{\prime}\left(n^{\prime}-1\right)\left(m^{\prime}+1\right)\right\}, \\
c\left(n, m ; n^{\prime}, m^{\prime}\right)= & \left\{n(n-1) n^{\prime}\left(n^{\prime}-1\right)\right. \\
& \left.\times(m+1)\left(m^{\prime}+1\right)\right\}^{1 / 2} .
\end{aligned}
$$

The differential equations in Eq. (24) can be recast into a set of differential matrix systems of the form

$$
\frac{d}{d \tau} \mathbf{P}\left(n, m ; n^{\prime}, m^{\prime}\right)=\boldsymbol{M}\left(n, m ; n^{\prime}, m^{\prime}\right) \mathbf{P}\left(n, m ; n^{\prime}, m^{\prime}\right) \text {, }
$$

with $\mathbf{P}\left(n, m ; n^{\prime}, m^{\prime}\right)$ being the column vector

$$
\mathbf{P}\left(n, m ; n^{\prime}, m^{\prime}\right)=\left(\begin{array}{c}
P_{n, m ; n^{\prime}, m^{\prime}} \\
P_{n+2, m-1 ; n^{\prime}+2, m^{\prime}-1} \\
\vdots \\
P_{n+2 k, m-k ; n^{\prime}+2 k, m^{\prime}-k} \\
\vdots
\end{array}\right),
$$

where $k=0,1, \ldots, \min \left(m, m^{\prime}\right)$ and $\boldsymbol{M}\left(n, m ; n^{\prime}, m^{\prime}\right)$ is the finite-dimensional band matrix (of width 2 )

$\boldsymbol{M}\left(n, m ; n^{\prime}, m^{\prime}\right)=\left(\begin{array}{cccccc}-b_{00} & b_{01} & & & \\ & -b_{11} & b_{12} & & 0 & \\ & & \ddots & \ddots & \\ & & & -b_{k k} & b_{k, k+1} \\ & & & & \ddots & \ddots\end{array}\right)$,

with

$$
\begin{aligned}
& b_{k k}=b\left(n+2 k, m-k ; n^{\prime}+2 k, m^{\prime}-k\right), \\
& b_{k-1, k}=c\left(n+2 k, m-k ; n^{\prime}+2 k, m^{\prime}-k\right) .
\end{aligned}
$$

The solution of Eq. (26) can be expressed in the form
$\mathbf{P}\left(n, m ; n^{\prime}, m^{\prime}\right)(\tau)$

$$
=\sum_{j=0}^{\min \left(m, m^{\prime}\right)} C_{j} \mathbf{A}_{j}\left(n, m ; n^{\prime}, m^{\prime}\right) \exp \left(\lambda_{j} \tau\right),
$$

where $\lambda_{j}=-b_{j j}$ are the eigenvalues (assumed simple) of the triangular matrix $\boldsymbol{M}\left(n, m ; n^{\prime}, m^{\prime}\right)$. The $\mathbf{A}_{j}\left(n, m ; n^{\prime}, m^{\prime}\right)$ are right eigenvectors whose components satisfy the recursion relations

$$
\begin{aligned}
-b_{k k} A_{j k}\left(n, m ; n^{\prime}, m^{\prime}\right)+b_{k, k+1} A_{j, k+1}\left(n, m ; n^{\prime}, m^{\prime}\right) \\
=-b_{j j} A_{j k}\left(n, m ; n^{\prime}, m^{\prime}\right) .
\end{aligned}
$$

From this relation it can be obtained by iteration that

$A_{j k}\left(n, m ; n^{\prime}, m^{\prime}\right)=\left\{\begin{array}{l}\prod_{t=k+1}^{j} \frac{b_{t-1, t}}{b_{t-1, t-1}-b_{j j}} \\ 1 \text { if } k=j \\ 0 \text { if } k>j .\end{array}\right.$

The left eigenvectors $\mathbf{B}_{j}\left(n, m ; n^{\prime}, m^{\prime}\right)$ are similarly introduced; they satisfy the recursion relations

$b_{k-1, k} B_{j, k-1}\left(n, m ; n^{\prime}, m^{\prime}\right)-b_{k k} B_{j k}\left(n, m ; n^{\prime}, m^{\prime}\right)$

$$
=-b_{j j} B_{j k}\left(n, m ; n^{\prime}, m^{\prime}\right) \text {. }
$$

Iteration allows us to obtain

$$
B_{j k}\left(n, m ; n^{\prime}, m^{\prime}\right)=\left\{\begin{array}{l}
0 \text { if } k<j \\
1 \text { if } k=j \\
\prod_{t=j+1}^{k} \frac{b_{k, k+1}}{b_{k+1, k+1}-b_{j j}} \text { if } k>j .
\end{array}\right.
$$

Forming the scalar product with $\mathbf{B}_{k}\left(n, m ; n^{\prime}, m^{\prime}\right)$ from the left in the two sides of Eq. (27) for $\tau=0$, the orthonormality condition

$$
\left(\mathbf{B}_{k}, \mathbf{A}_{j}\right)=\sum_{l} B_{k l} A_{j l}=\delta_{k j}
$$

leads to

$$
C_{j}=\sum_{h} B_{j h}\left(n, m ; n^{\prime}, m^{\prime}\right) P_{n+2 h, m-h ; n^{\prime}+2 h, m^{\prime}-h}(0) .
$$

Substituting Eq. (30) into Eq. (27), we get

$$
\begin{aligned}
P_{n+2 k, m-k ; n^{\prime}+2 k, m^{\prime}-k}(\tau) & \\
=\sum_{j} \sum_{h} & B_{j h}\left(n, m ; n^{\prime}, m^{\prime}\right) A_{j k}\left(n, m ; n^{\prime}, m^{\prime}\right) \\
& \times \exp \left(-b_{j j} \tau\right) P_{n+2 h, m-h ; n^{\prime}+2 h, m^{\prime}-h}(0),
\end{aligned}
$$

where $j, h=0,1, \ldots, \min \left(m, m^{\prime}\right)$. Taking $k=0$ in the last formula and inserting in it the expressions Eqs. (28) and (29) for the coefficients $A_{j k}$ and $B_{j h}$, we obtain the final expression for the off-diagonal matrix elements: 


$$
\begin{aligned}
P_{n, m ; n^{\prime}, m^{\prime}}(\tau)= & \sum_{j=0}^{\min \left(m, m^{\prime}\right)} \sum_{h=j}^{\min \left(m, m^{\prime}\right)} \frac{\prod_{t=1}^{h} c\left(n+2 t, m-t ; n^{\prime}+2 t, m^{\prime}-t\right)}{\prod_{t=0}^{h}\left[b\left(n+2 t, m-t ; n^{\prime}+2 t, m^{\prime}-t\right)-b\left(n+2 j, m-j ; n^{\prime}+2 j, m^{\prime}-j\right)\right]} \\
& \times \exp \left[-b\left(n+2 j, m-j ; n^{\prime}+2 j, m^{\prime}-j\right) \tau\right] P_{n+2 h, m-h ; n^{\prime}+2 h, m^{\prime}-h}(0) .
\end{aligned}
$$

In the degenerate situation, i.e., when there are multiple eigenvalues in the matrix $\boldsymbol{M}\left(n, m ; n^{\prime}, m^{\prime}\right)$ diagonal, there will appear singularities in the rhs of Eq. (31). In that case it should be still possible to recover this formula by adding some appropriate renormalization prescription.

\section{DISCUSSION}

We show in Figs. 3-7 the change, in time, of the statistical properties of the photon fields in several cases of interest, obtained by computer calculation and taking as initial conditions the coherent or chaotic distributions of photon-number probability for each of the two radiation modes involved. As is shown in Fig. 3, the initial rate in the growing of the Stokes mode intensity is greater in the case where, initially, the incident pumping mode is chaotic. Figure 6 shows the change in time of the second-order coherence degree $g_{m}^{(2)}$. We note [see Fig. 6(a)] that at time zero, there is a sudden increase in its value; this increase is quite more apparent for initially chaotic pumping light in which case $g_{m}^{(2)}$ tends to the completely incoherent value for the Stokes mode. In both cases, after the most incoherent situation, $g_{m}^{(2)}$ slowly decreases, but whereas curve $a$ tends to recover the initial completely coherent situation, curve $b$ tends to stabilize at a value near 1.5.

The behavior in time of $g_{m}^{(2)}$ for the chaotic Stokes mode is shown in Fig. 6(b). It can be noticed that, for

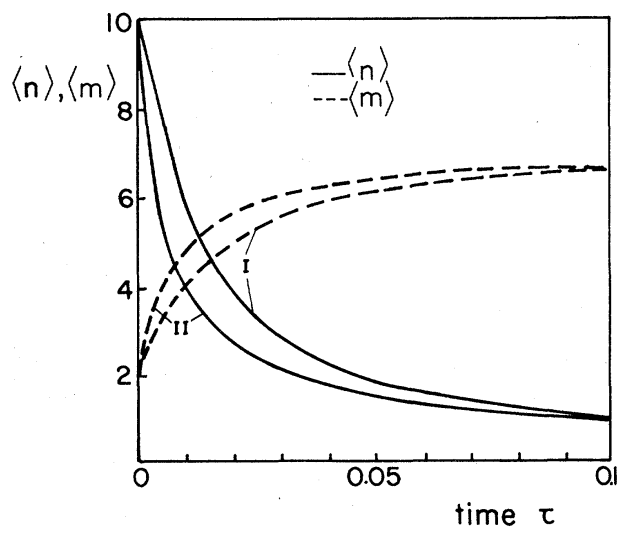

FIG. 3. Average number of photons $\langle n\rangle$ (incident field) and $\langle m\rangle$ (Stokes field) vs time, with $\left\langle n_{0}\right\rangle=10,\left\langle m_{0}\right\rangle=2$. I, initially coherent incident field, coherent Stokes field; II, chaotic incident field-coherent Stokes field. chaotic input photons, $g_{m}^{(2)}$ increases initially up to values significantly higher than its mutual chaotic value which is a typical enhanced photon-bunching phenomenon. The second-order coherence degree $g_{n}^{(2)}$ (see Fig. 5) tends to decrease as time progresses, owing to the fact that its aver-
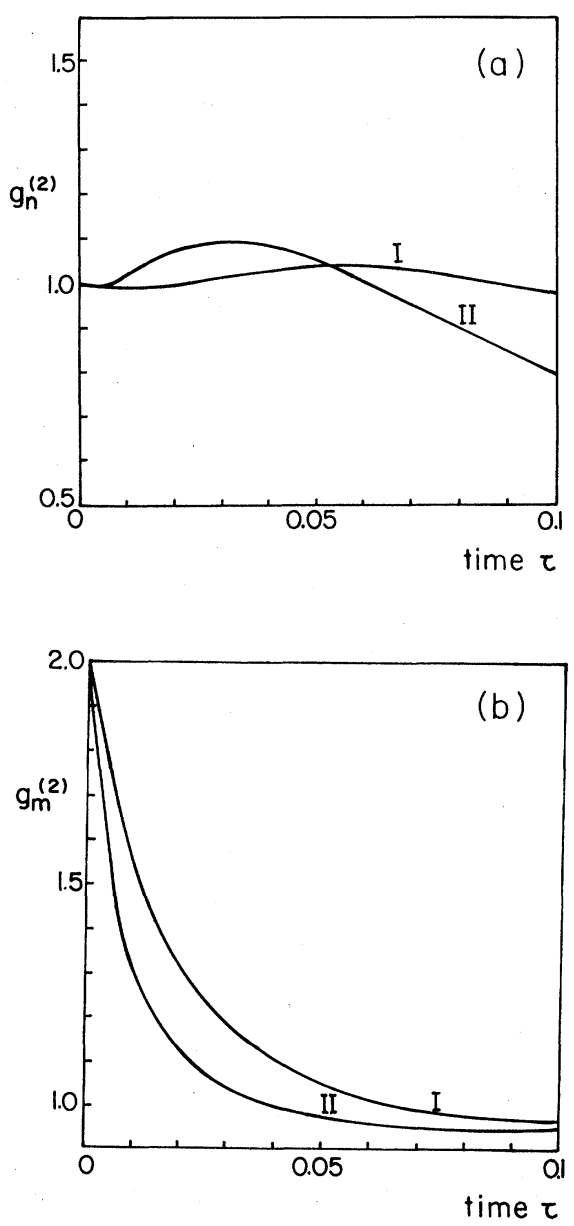

FIG. 4. (a) Second-order coherence degree $g_{n}^{(2)}$ of the incident field vs time for initially coherent incident field. I, initial average number of photons in the incident field $\left\langle n_{0}\right\rangle=10$; II, $\left\langle n_{0}\right\rangle=15$. In both cases $\left\langle m_{0}\right\rangle=0$. (b) Second-order coherence degree $g_{m}^{(2)}$ of the Stokes field vs time for initially coherent incident field. I, $\left\langle n_{0}\right\rangle=0$; II, $\left\langle n_{0}\right\rangle=15$. In both cases $\left\langle m_{0}\right\rangle=0$. 

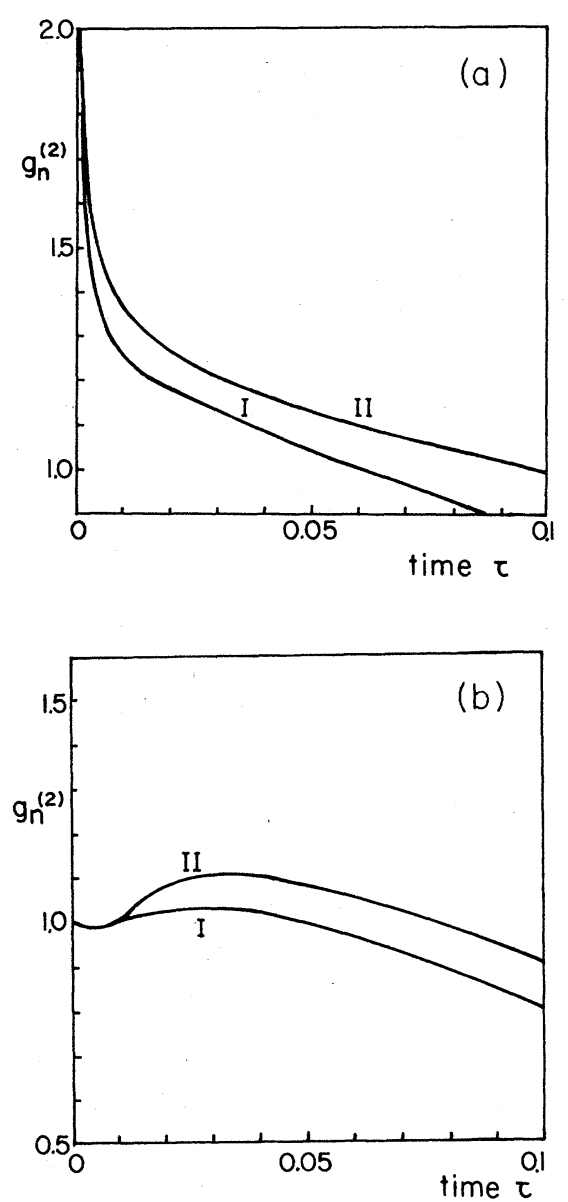

FIG. 5. (a) Second-order coherence degree $g_{n}^{(2)}$ of the incident field vs time for initially chaotic incident field. I, initially coherent Stokes field; II, initially chaotic Stokes field. In both cases $\left\langle n_{0}\right\rangle=10,\left\langle m_{0}\right\rangle=2$. (b) Second-order coherence degree $g_{n}^{(2)}$ of the incident field vs time for initially coherent incident field. I, initially coherent Stokes field; II, initially chaotic Stokes field. In both cases $\left\langle n_{0}\right\rangle=10,\left\langle m_{0}\right\rangle=2$.

age photon number decays to almost zero for large times. Photon antibunching, ${ }^{8,9}$ i.e., $g_{n}^{(2)}<1$, is observed to occur in all cases for the incident light.

Another statistical function of interest is the mutual correlation degree $g_{c}$. In Fig. 7 we observe the positive correlation at initial times when the incident mode is initially chaotic. This reflects the fact that in this case the Stokes photons tend to emerge correlated with the positive fluctuations in the average number of photons present in the chaotic incident beam.

Besides photon antibunching there is another nonclassical effect revealed by the violation of the condition

$$
g_{c}^{2} \leq g_{n}^{(2)} g_{m}^{(2)},
$$

which is the quantum translation of the Cauchy inequality. ${ }^{10}$ This violation means that the beams have acquired a larger mutual correlation than the correlation between photons in the same beam, and has been already discussed
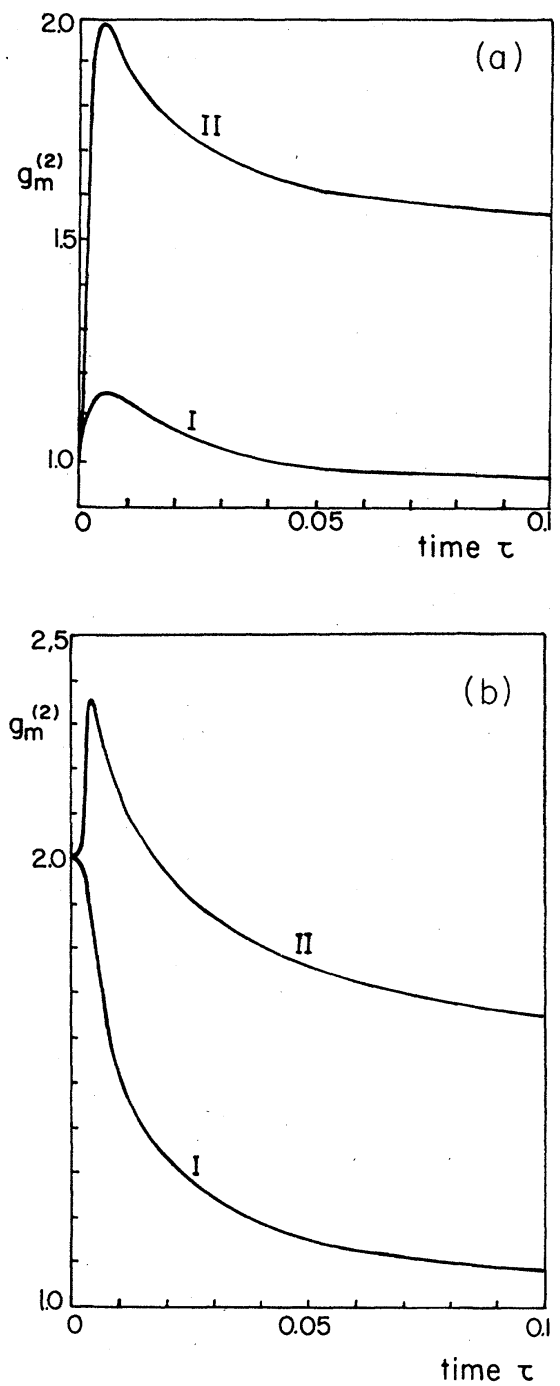

FIG. 6. (a) Second-order coherence degree $g_{m}^{(2)}$ of the Stokes field vs time for initially coherent Stokes field. I, initially coherent incident field; II, initially chaotic incident field. In both cases $\left\langle n_{0}\right\rangle=10,\left\langle m_{0}\right\rangle=2$. (b) Second-order coherence degree $g_{m}^{(2)}$ of the Stokes field vs time for initially chaotic Stokes field. I, initially coherent incident field; II, initially chaotic incident field. In both cases $\left\langle n_{0}\right\rangle=10,\left\langle m_{0}\right\rangle=2$.

by Zubairy ${ }^{11}$ for the two-photon laser statistics and actually observed to occur in some cases. ${ }^{12}$ Figure 8 shows that, at sufficiently large times, the value of $K=g_{c}^{2} / g_{n}^{(2)} g_{m}^{(2)}$ becomes greater than 1 , so that the Cauchy inequality is eventually violated in the cases under study. This fact should be related with the ultimate loss of coherence and with the appearance of photon antibunching as the incident beam becomes exhausted; in fact $g_{n}^{(2)} \rightarrow 0$ as $\tau \rightarrow \infty$, while $g_{c}$ and $g_{m}^{(2)}$ remain finite. The low number of photons involved makes it, however, hazardous to claim for the physical realization of such nonclassical states of radiation. 


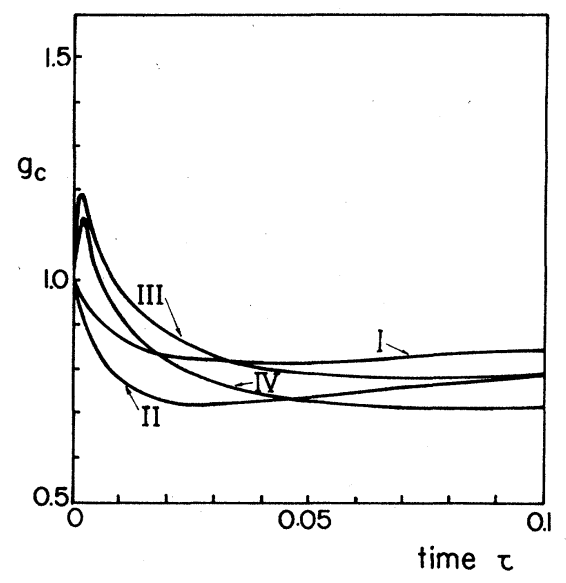

FIG. 7. Cross-correlation degree $g_{c}$ vs time for initially I, coherent incident field, coherent Stokes field; II, coherent incident field, chaotic Stokes field; III, chaotic incident field, coherent Stokes field; IV, chaotic incident field, chaotic Stokes field. In all cases $\left\langle n_{0}\right\rangle=10,\left\langle m_{0}\right\rangle=2$.

Figures 9 and 10, show the behavior of some statistical properties of radiation, when the Cauchy inequality starts to be violated, i.e., at threshold times $\tau_{\mathrm{th}}$, for the two beams in the initially coherent state. A linear progression of the remanent intensity $\left\langle n\left(\tau_{\text {th }}\right)\right\rangle$ with the initial intensity $\left\langle n_{0}\right\rangle$ is observed in Fig. 9. For the range of the $\left\langle n_{0}\right\rangle$ values considered here $\left(\left\langle n_{0}\right\rangle \leq 65\right)$, it can therefore be ascertained that about $95 \%$ of the initial photon energy should be absorbed in the incident beam before these quantum-statistical effects emerge. It should be noticed also that $K$ increases above its initial value for an initially chaotic incident beam, i.e., $(d K / d \tau)_{\tau=0}>0$ [see Eqs. (14)]. This, however, is not expected to be but a slight enhancement in the relative mutual correlation at the early stage of the process, with no further violation of the Cauchy inequality. Finally, the quasilogarithmic variation of $\tau_{\text {th }}$ versus $\left\langle n_{0}\right\rangle$ shown in Fig. 10 indicates that the higher the initial intensity, the sooner the discussed quantum effect is manifested.

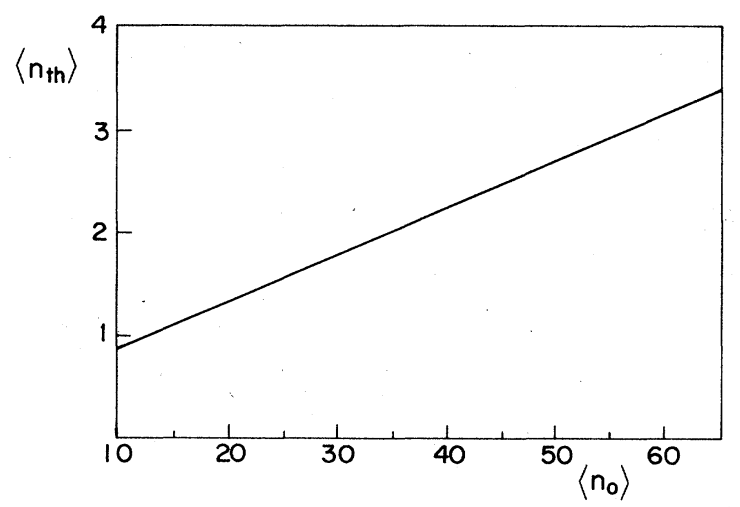

FIG. 9. Average number of photons in the incident beam at threshold time $\left\langle n_{\text {th }}\right\rangle$ vs initial average number of photons $\left\langle n_{0}\right\rangle$. The two beams are initially in coherent states and $\left\langle m_{0}\right\rangle=2$.

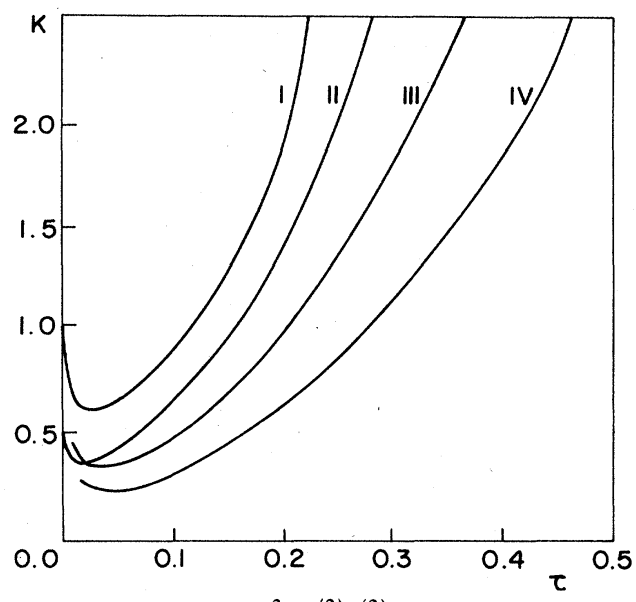

FIG. 8. Quotient $K=g_{c}^{2} / g_{n}^{(2)} g_{m}^{(2)}$ vs time for initially I, coherent incident field, coherent Stokes field; II, coherent incident field, chaotic Stokes field; III, chaotic incident field, coherent Stokes field; IV, chaotic incident field, chaotic Stokes field. In all cases $\left\langle n_{0}\right\rangle=10,\left\langle m_{0}\right\rangle=2$.

\section{APPENDIX}

In order to find the constants $C_{j}(I)$ ( $I$ corresponding to a nondegenerate chain), we use the eigenvectors "at left" (row vectors) corresponding to the matrix $\boldsymbol{M}^{(I)}$ defined by the relation

$$
\mathbf{B}_{j}(I) \boldsymbol{M}^{(I)}=-b_{j}(I) \mathbf{B}_{j}(I), j=0,1, \ldots, \alpha(I) .
$$

Solving the corresponding linear equations, we get

$$
B_{j m}(I)=\left\{\begin{array}{l}
1 \text { if } m=0 \\
0 \text { if } m>j \\
\prod_{k=0}^{m-1} \frac{b_{k}(I)-b_{j}(I)}{b_{k}(I)} \text { if } m<j .
\end{array}\right.
$$

It can be shown that eigenvectors $\mathbf{A}_{j}$ and $\mathbf{B}_{j}$ satisfy the orthogonality condition,

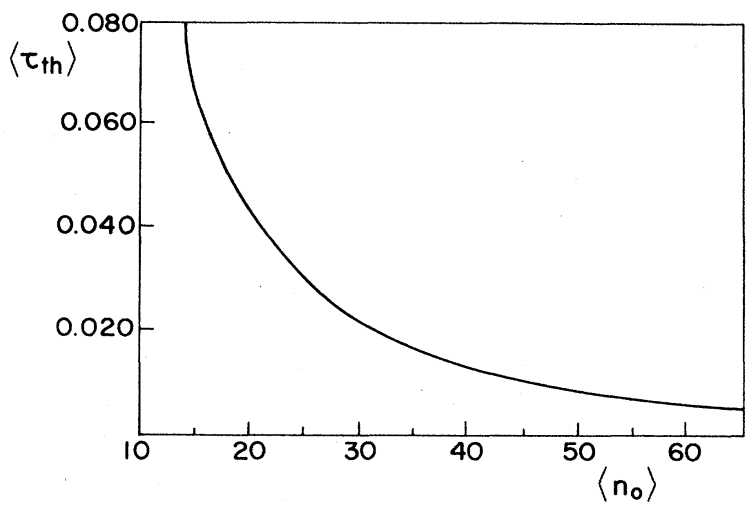

FIG. 10. Threshold time $\tau_{\text {th }}$ when $K=1$ vs initial average number of photons $\left\langle n_{0}\right\rangle$. The two beams are initially in coherent states and $\left\langle m_{0}\right\rangle=2$. 


$$
\left(\mathbf{B}_{k}, \mathbf{A}_{j}\right)=\sum_{m} B_{k m} A_{j m}=0 \text { if } j \neq k .
$$

The constants $C_{j}$ can be calculated now by inversion of the identity equation,

$$
\mathbf{P}^{(I)}(0)=\sum_{j=0}^{\alpha(I)} C_{j}(I) \mathbf{A}_{j}(I) .
$$

We multiply this last equation to the left by eigenvectors $\mathbf{B}_{k}(I)$ and obtain finally

$$
C_{k}(I)=\frac{\left(\mathrm{B}_{k}(I), P^{(I)}(0)\right)}{\left(\mathrm{B}_{k}(I), A_{k}(I)\right)}=\frac{\sum_{m} B_{k m} P_{m}^{(I)}(0)}{\sum_{m} B_{k m} A_{k m}} .
$$

For the case of degenerate chains, we can use the above formulas for the constants $C_{j}$ only for the case of simple eigenvalues, i.e., for $j \neq g, h$. For the degenerate eigenvalues $\lambda=-b(I)$, we first obtain the vector $\mathbf{D}(I)$ defined by the relation

$$
\left[\boldsymbol{M}^{(I)}+\mathbb{1} b(I)\right] \mathbf{D}(I)=\mathbf{A}_{h}(I),
$$

where $\mathbf{A}_{h}$ is the sole existing eigenvector, corresponding to the eigenvalue $-b(I)$. If we solve the corresponding linear-algebraic equations we obtain the recursion relation for the components of $\mathbf{D}$,

$D_{m}(I)=\frac{A_{h, m+1}(I)-\left[b(I)-b_{m+1}(I)\right] D_{m+1}(I)}{b_{m}(I)}$.

$$
\text { Determination of the constants } \iota(I) \text { and } \kappa(I)
$$

We start by calculating the left eigenvectors $\mathbf{B}_{j}(I)$ and $\mathbf{B}_{h}(I)(j \neq g, h)$ with the aid of the above formulas [see (A2)]. The constants $C_{j}(I)$ for $j \neq g, h$ can be taken as above. Now we construct an auxiliary row vector $\mathbf{E}^{T}(I)$ satisfying

$$
\mathbf{E}^{T}(I)\left[\boldsymbol{M}^{(I)}+\mathbb{1} b(I)\right]=\mathbf{B}_{h}(I) .
$$

It can easily be shown that the components $E_{m}$ satisfy the recursion relation

$$
E_{m+1}=\frac{B_{h m}-\left[b(I)-b_{m}(I)\right] E_{m}}{b_{m}(I)} .
$$

Also, the following orthogonality relations are easily obtained:

$$
\begin{aligned}
& \left(\mathbf{B}_{k}, \mathbf{A}_{j}\right)=0, \quad j \neq k, j, k \neq g, h, \\
& \left(\mathbf{B}_{k}, \mathbf{A}_{h}\right)=0, \quad k \neq g, h, \\
& \left(\mathbf{B}_{h}, \mathbf{A}_{k}\right)=0, \quad k \neq g, h, \\
& \left(\mathbf{E}^{T}, \mathbf{A}_{j}\right)=0, \quad j \neq g, h, \\
& \left(\mathbf{B}_{j}, \mathbf{D}\right)=0, \quad j \neq g, h, \\
& \left(\mathbf{E}^{T}, \mathbf{A}_{h}\right)-\left(\mathbf{B}_{h}, \mathbf{D}\right)=0, \\
& \left(\mathbf{B}_{h}, \mathbf{A}_{h}\right)=0 .
\end{aligned}
$$

Now we write the identity equation, valid for degenerate chains

$\mathbf{P}^{(I)}(0)=\sum_{\substack{j=0 \\ j \neq g, h}}^{\alpha(I)} C_{j}(I) \mathbf{A}_{j}(I)+\kappa(I) \mathbf{A}_{h}(I)+\iota(I) \mathbf{D}(I)$

and multiply to the left by the row vectors $B_{k}(k \neq g, h)$, $B_{h}$, and $E$, respectively. Using the above orthogonality relations we obtain the equations

$$
\begin{aligned}
& \left(\mathbf{B}_{k}, \mathbf{P}^{(I)}(0)\right)=C_{k}\left(\mathbf{B}_{k}, \mathbf{A}_{k}\right), \\
& \left(\mathbf{B}_{h}, \mathbf{P}^{(I)}(0)\right)=\kappa\left(\mathbf{B}_{h}, \mathbf{A}_{h}\right)+\iota\left(\mathbf{B}_{h}, \mathbf{D}\right), \\
& \left(\mathbf{E}^{T}, \mathbf{P}^{(I)}(0)\right)=\kappa\left(\mathbf{E}^{T}, \mathbf{A}_{h}\right)+\iota\left(\mathbf{E}^{T}, \mathbf{D}\right) .
\end{aligned}
$$

The first of these equations is directly solved,

$$
C_{k}=\frac{\left(\mathbf{B}_{k}, \mathbf{P}^{(I)}(0)\right)}{\left(\mathbf{B}_{k}, \mathbf{A}_{k}\right)}, k \neq g, h
$$

The last two equations form an easy solvable system: we find, after some algebraic manipulations,

$$
\kappa=\frac{\left(X_{1} Z-X_{2} Z\right)}{X^{*}}, \iota=\frac{\left(X_{4} Y-X_{3} Z\right)}{X^{*}},
$$

where $\quad X_{1}=\left(\mathbf{B}_{h}, \mathbf{D}\right), \quad X_{2}=\left(\mathbf{E}^{T}, \mathbf{D}\right), \quad X_{3}=\left(\mathbf{B}_{h}, \mathbf{A}_{h}\right)$, $X_{4}=\left(\mathbf{E}^{T}, \mathbf{A}_{h}\right), \quad Y=\left(\mathbf{B}_{h}, \mathbf{P}^{(I)}(0)\right), \quad Z=\left(\mathbf{E}^{T}, \mathbf{P}^{(I)}(0)\right), \quad$ and $X^{*}=X_{1} X_{4}-X_{2} X_{3}$. Noting that $X_{3}$ is identically zero [see Eq. (A8g)], we finally obtain

$$
\kappa=\frac{\left(X_{1} Z-X_{2} Y\right)}{X_{1} X_{4}}, \iota=Y / X_{1} \text {. }
$$

${ }^{1}$ H. D. Simaan, J. Phys. A 8, 1620 (1975).

${ }^{2}$ D. C. Hanna, M. A. Yuratich, and D. Cotter, in Nonlinear Optics of Free Atoms and Molecules, edited by D. L. MacAdam (Springer, Berlin, 1977), p. 239; M. Schubert and B. Wilhelmi, in Progress in Optics, edited by E. Wolf (North-Holland, Amsterdam, 1980), Vol. 17, p. 165.

${ }^{3}$ H. D. Simaan, J. Phys. A 11, 1799 (1978).

${ }^{4}$ M. S. Zubairy and J. J. Yeh, Phys. Rev. A 21, 1624 (1980).

5Y. R. Shen, Phys. Rev. 155, 921 (1967).

${ }^{6}$ M. O. Scully and K. G. Whitney, in Progress in Optics, edited by E. Wolf (North-Holland, Amsterdam, 1972), Vol. 10, p. 89.

${ }^{7} \mathrm{R}$. Loudon, The Quantum Theory of Light (Oxford University, New York, 1973), p. 324.

${ }^{8}$ R. Loudon, Rep. Prog. Phys. 43, 913 (1980).

${ }^{9}$ H. Paul, Rev. Mod. Phys. 54, 1061 (1982)

${ }^{10}$ See Ref. 8

${ }^{11}$ M. S. Zubairy, Phys. Lett. 87A, 162 (1982).

12J. F. Clauser, Phys. Rev. D 9, 853 (1974). 